\title{
Comparison of Ontology-Based Semantic-Similarity Measures in the Biomedical Text
}

\author{
Ahmad Fayez S. Althobaiti \\ Department of Computer and Information Sciences, Al-Imam Muhammad Ibn Saud Islamic University, Riyadh, \\ Saudi Arabia \\ Email: Afalthobaiti@imamu.edu.sa
}

How to cite this paper: Althobaiti, A.F.S. (2017) Comparison of Ontology-Based Semantic-Similarity Measures in the Biomedical Text. Journal of Computer and Communications, 5, 17-27. https://doi.org/10.4236/jcc.2017.52003

Received: January 17, 2017

Accepted: February 6, 2017

Published: February 9, 2017

Copyright $\odot 2017$ by author and Scientific Research Publishing Inc. This work is licensed under the Creative Commons Attribution International License (CC BY 4.0).

http://creativecommons.org/licenses/by/4.0/ (c) (i) Open Access

\begin{abstract}
In recent years, there are many types of semantic similarity measures, which are used to measure the similarity between two concepts. It is necessary to define the differences between the measures, performance, and evaluations. The major contribution of this paper is to choose the best measure among different similarity measures that give us good result with less error rate. The experiment was done on a taxonomy built to measure the semantic distance between two concepts in the health domain, which are represented as nodes in the taxonomy. Similarity measures methods were evaluated relative to human experts' ratings. Our experiment was applied on the ICD10 taxonomy to determine the similarity value between two concepts. The similarity between 30 pairs of the health domains has been evaluated using different types of semantic similarity measures equations. The experimental results discussed in this paper have shown that the Hoa A. Nguyen and Hisham Al-Mubaid measure has achieved high matching score by the expert's judgment.
\end{abstract}

\section{Keywords}

Semantic Similarity Measure, Structure-Based Measures, Edge-Counting, Feature-Based Measures, Hybrid Measures, ICD-10, MeSH Ontology

\section{Introduction}

Semantic similarity between concepts is a method to measure the semantic similarity or the semantic distance between two concepts according to a given ontology. The semantic similarity measuring techniques can be classified into three classes as follows. The first measure semantic similarity by using ontology or taxonomy (e.g. Word Net, UMLS/ICD10) to calculate the distance between the 
concept nodes in the ontology tree or hierarchy [1]. The second class of techniques uses training corpora and information content (IC) to estimate the semantic similarity and relatedness between two concepts. The third class simply includes the techniques that employ a combination from the first two classes. Measures of semantic similarity and relatedness are used in applications such as information extraction and retrieval, classification and ranking, detection of redundancy, detection and correction of malapropisms. In this paper, we analyze an ontology-based semantic similarity measure and apply it in the biomedical domain, using ICD10 as knowledge sources.

\section{Background and Related Work}

\subsection{UMLS and ICD10}

The Unified Medical Language System (UMLS) project started at the National Library of Medicine (NLM) in 1986.It consists of three main knowledge sources: first: Metathesaurus consists of more than 1 million biomedical concepts from over 130 sources and supports 17 languages. Second: Semantic Network contains 135 broach categories and 54 relationships between categories. Third: SPECIALIST Lexicon \& Lexical Tools includes lexical information and programs for processing language [2]. In Metathesaurus of UMLS 2005AB (June 2005), there are 133 source vocabularies classified into 73 families. They have multiple translations (e.g., MeSH, ICPC, and ICD-10) and have many variants (American-British equivalents, Australian extension/adaptation) [2].

ICD10 stands for International Classification Diseases $10^{\text {th }}$ revision: An international standard used to classify diseases and other health problems adopted by World Health Organization (WHO) [3]. The newest edition (ICD-10) is divided into 21 chapters: (Infections, Neoplasm, Blood Diseases, Endocrine Diseases, etc.), and denote about 14,000 classes of diseases and related problems.

\subsection{ICD10 Taxonomy}

The first character of the ICD code is a letter, and each letter is associated with a particular chapter, except for the letter D, which is used in both Chapter II, Neoplasm, and Chapter III, Diseases of the blood and blood-forming organs and certain disorders involving the immune mechanism, and the letter $\mathrm{H}$, which is used in both Chapter VII, Diseases of the eye and adnexa and Chapter VIII, Diseases of the ear and mastoid process. Four chapters (Chapters I, II, XIX, and XX) use more than one letter in the first position of their codes. Each chapter contains sufficient three-character categories to cover its content; not all available codes are used, allowing space for future revision and expansion. Chapters I-XVII relate to diseases and other morbid conditions, and Chapter XIX to injuries, poisoning and certain other consequences of external causes. The remaining chapters complete the range of subject matter nowadays included in diagnostic data. Chapter XVIII covers Symptoms, signs and abnormal clinical and laboratory findings, not elsewhere classified. Chapter XX, External causes of 
morbidity and mortality, was traditionally used to classify causes of injury and poisoning, but, since the Ninth Revision, has also provided for any recorded external cause of diseases and other morbid conditions. Finally, Chapter XXI, Factors influencing health status and contact with health services, is intended for the classification of data explaining the reason for contact with health-care services of a person not currently sick, or the circumstances in which the patient is receiving care at that particular time or otherwise having some bearing on that person's care. The chapters are subdivided into homogeneous "blocks" of three-character categories. Most of the three character categories are subdivided by means of a fourth, numeric character after a decimal point, allowing up to 10 subcategories [3]. We have used the following taxonomy in order to measure the similarity between the health domain types which is being represented as nodes in the taxonomy. In our experiment, the similarity is measured using different types of semantic measures. From the evaluation result, the best measure will be used in our benchmark model. Figure 1 below describes ICD10 nodes:

\subsection{Related Work}

Several methods and a lot of research work for determining semantic similarity measures have been proposed in the last few decades, the similarity between two concepts/nodes is also called as relatedness. To measure the relatedness of two concepts $\mathrm{C}_{1}$ and $\mathrm{C}_{2}$, researchers have used many methods in these two approaches that can be classified into two categories: the first one is based on the graph, using distance concept's that mainly considers the lengths of the paths connecting the concepts.

Rada [5] defined the conceptual distance between two words in the "is-a" hierarchy relationships as the length of the shortest path connecting the two

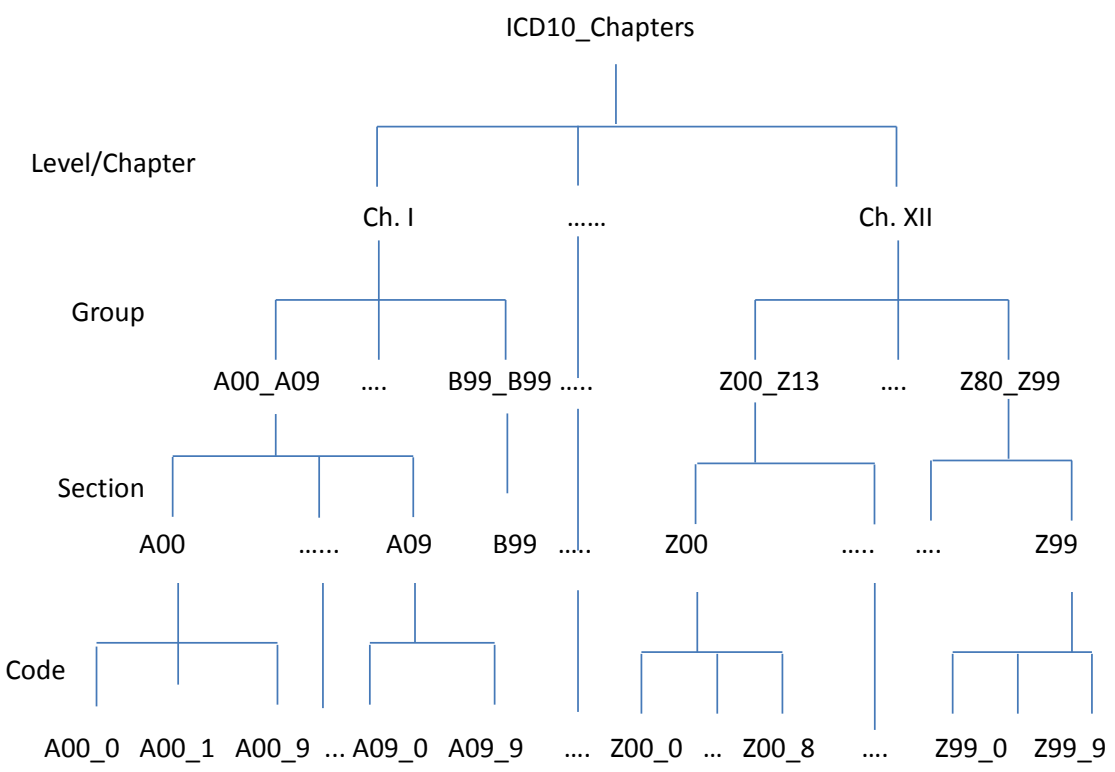

Figure 1. Fragment of the ICD-10 taxonomy [4]. 
words. In this measure the semantic distance is computed by counting the number of edges between concepts in the taxonomy. The experiments were conducted using MeSH (Medical Subject Headings-Biomedical ontology) ontology.

Resnik [6] is the first person who employed Information content to calculate semantic similarity in the "IS-A" hierarchy relationship. He proposed that the more information content two words share, the more similar they are. Following Resnik's method, several other measures were proposed.

Lin [7] the authors of this work have proposed a measure based on an ontology restricted to hierarchic links and a corpus. This similarity takes into account the information shared by two concepts like Resnik, but the difference between them is in the definition. The definition contains the same components as Resnik measure but the combination is not a difference.

\section{Semantic Similarity Measures for Single Ontology}

In this paper, we focus only on these semantic similarity measures that used ontology as a primary information source. The main semantic measures could be classified into Structure-based measures and Information content (IC) measures:

\subsection{Ontology Structure-Based Similarity Measures}

Most of the measures that are based on the structure of the ontology are actually based on: path length/distance (shortest path length) between the two concept nodes, and depth of concept nodes in the ontology/is-a hierarchy tree, e.g. some of the measures are based on Word Net include: path length, Wu \& palmer, Leacock \& Chodorow, Resink, and Lin et al. [8] [9].

\subsubsection{Path Length Based Measures (Shortest Path)}

In this method, the similarity measurement among concepts is determined according to the path distance, which separates the concepts on the taxonomy or ontology structure. In this measure the distance between two concepts $\mathrm{C} 1, \mathrm{C} 2$ is computed as the shortest path linking them as estimate distance.

$$
\text { distRada }=\mathrm{SP}(\mathrm{C} 1, \mathrm{C} 2)
$$

Also, asimple edge-counting measure proposed by Rada [5]:

$$
\operatorname{disRada}(\mathrm{c} 1, \mathrm{c} 2)=\mathrm{N} 1+\mathrm{N} 2
$$

where:

$\mathrm{N} 1$ and $\mathrm{N} 2$ are the minimum numbers of taxonomical links from $\mathrm{c} 1$ to $\mathrm{c} 2$ to their LCS, respectively. The similarities between two concepts $\mathrm{C} 1$ and C2 can be formulated as follows:

$$
\operatorname{Sim}(\mathrm{c} 1, \mathrm{c} 2)=2 * \operatorname{Max}-\text { length }(\mathrm{c} 1, \mathrm{c} 2)
$$

where:

Max is the maximum depth of the taxonomy.

Length $(\mathrm{c} 1, \mathrm{c} 2)$ is the shortest path length $\mathrm{C} 1$ and $\mathrm{C} 2$. 


\subsubsection{Wu and Palmer Measure}

This similarity measure considers the position of $\mathrm{C} 1$ and $\mathrm{C} 2$ to the position of the most specific common concept C. Several parents can be shared by $\mathrm{C} 1$ and $\mathrm{C} 2$ by multiple paths. The most specific common concept is the closest common ancestor $\mathrm{C}$ (the common parent related with the minimum number of is-a links with concepts $\mathrm{C} 1$ and $\mathrm{C} 2$ ) [10].

$$
\operatorname{Sim}(\mathrm{c} 1, \mathrm{c} 2)=\frac{2 * \mathrm{~N} 3}{\mathrm{~N} 1+\mathrm{N} 2+2 * \mathrm{~N} 3}
$$

where:

$\mathrm{N} 1$ and $\mathrm{N} 2$ are the distance from the specific common concept to concept $\mathrm{C} 1$ and $\mathrm{C} 2$ respectively. $\mathrm{N} 3$ is the depth of the least common subsumer (The least common subsumer, $\mathrm{LCS}(\mathrm{C} 1, \mathrm{C} 2)$, of two concept nodes $\mathrm{C} 1$ and $\mathrm{C} 2$ are the lowest nodes that can be a parent for $\mathrm{C} 1$ and $\mathrm{C} 2$. For example, in Figure 1, (LCS $(\mathrm{A} 00.0, \mathrm{~A} 00.9)=\mathrm{A} 00$ and LCS $(\mathrm{A} 00.0, \mathrm{~A} 09.0)=\mathrm{A} 00-\mathrm{A} 09)$ of two concepts nodes, and N1, N2 are the path lengths from each concept node to LCS, respectively. From our taxonomy (Figure 1), we can calculate the similarity between concepts $\mathrm{C}_{1}$ and $\mathrm{C}_{2}$ as following:

$$
\text { Similarity }(\text { A00.0, A09.0 })=\frac{2 * 2}{2+2+(2 * 2)}=0.50 \text {. }
$$

\subsubsection{Leacock and Chodorow Measure}

In this method, the similarity between two concepts is determined by discovering the shortest path length, which connects these two concepts in the taxonomy/ontology. The similarity is calculated as the negative algorithm of this value. The similarities between two concepts $\mathrm{C} 1$ and $\mathrm{C} 2$ can be formulated as follows [6]:

$$
\operatorname{Sim}_{\mathrm{LC}}(\mathrm{C} 1, \mathrm{C} 2)=-\log \log \left(\frac{\mathrm{sp}(\mathrm{c} 1, \mathrm{c} 2)}{2\left(\text { max_depth }_{-}\right)}\right)
$$

max_depth is longest of the shortest path linking concept to concept, which subsumed all others.

From our taxonomy (Figure 1), we can calculate the similarity between concepts $\mathrm{C}_{1}$ and $\mathrm{C}_{2}$ as following:

$$
\text { Similarity }(\text { A00.0, A09.0 })=-\log \log \left(\frac{4}{2(5)}\right)=0.3979400086 \text {. }
$$

\subsection{Information Content (IC) Measures}

Following is the standard argumentation of information theory [Ross, 1976], the information content of a concept $\mathrm{c}$ can be quantified as the negative $\log$ like lihood [11] [12].

$$
\mathrm{IC}(\mathrm{c})=-\log \log \mathrm{p}(\mathrm{c})
$$

From our taxonomy (Figure 1), we can calculate the similarity between concepts $\mathrm{C}_{1}$ and $\mathrm{C}_{2}$ as following: 


$$
\mathrm{IC}(\mathrm{A} 00-\mathrm{A} 09)=\frac{\log \log (\operatorname{depth}(\mathrm{C}))}{\log \log \left(\operatorname{deep}_{\max }\right)}=\log \log \frac{(2)}{\log \log (5)}=0.43067655807 .
$$

\subsubsection{Resink Measure}

In this measure, the similarity of two concepts $(\mathrm{c} 1, \mathrm{c} 2)$ is defined as the Information Content (IC) of their LCS, as shown in the following Equation (7):

$$
\operatorname{Sim} \operatorname{Res}(\mathrm{C} 1, \mathrm{C} 2)=-\log \mathrm{p}(\operatorname{LCS}(\mathrm{C} 1, \mathrm{C} 2))=\operatorname{IC}(\operatorname{LCS}(\mathrm{C} 1, \mathrm{C} 2))
$$

Where:

$$
\mathrm{IC}(\mathrm{C})=\frac{\log (\operatorname{depth}(\mathrm{C}))}{\log \log \left(\text { deep }_{\max }\right)}
$$

From our taxonomy (Figure 1), we can calculate the similarity between concepts $\mathrm{C}_{1}$ and $\mathrm{C}_{2}$ as following:

$$
\text { LCS }(\text { A00.0, A00.9) = A00 therefore Simres }(\text { A00.0, A00.9) }=\text { IC (A00) }
$$

Then:

$$
\operatorname{IC}(\mathrm{A} 00)=\frac{\log \log (\operatorname{depth}(C))}{\log \log \left(\text { deep }_{\max }\right)}=\log \log \frac{(3)}{\log \log (5)}=0.68260619448 .
$$

\subsubsection{Lin Similarity Measure}

This measure depends on the relation between information content (IC) of the LCS of two concepts and the sum of the information content of the individual concepts [7] [13].

$$
\operatorname{Simlin}(\mathrm{C} 1, \mathrm{C} 2)=\frac{2 * \operatorname{simres}(\mathrm{c} 1, \mathrm{c} 2)}{\mathrm{IC}(\mathrm{C} 1)+\mathrm{IC}(\mathrm{C} 2)}
$$

From our taxonomy (Figure 1), we can calculate the similarity between concepts $\mathrm{C}_{1}$ and $\mathrm{C}_{2}$ as following:

$$
\operatorname{Sim}_{\text {Lin }}(\mathrm{A} 00.0, \mathrm{~A} 09.0)=2 * /(1+1)=68 .
$$

\subsection{Semantic Similarity in the Biomedical Domain}

\subsubsection{Rada Measure}

Rada et al. [5] Proposed semantic distance as a potential measure of semantic similarity between two concepts in $\mathrm{MeSH}$, and implemented the shortest path length measure, called CDist, based on the shortest distance between two concept nodes in the ontology. They evaluated CDist on UMLS Metathesaurus (MeSH, SNOM-ED, ICD9), and compared the CDist similarity scores to human expert scores by correlation coefficients.

\subsubsection{Pedersen Measure}

Pedersen et al. [1] Proposed semantic similarity and relatedness in the biomedicine domain, by applied a corpus-based context vector approach to measuring thesimilarity between concepts in SNOMED-CT. Their context vector approach is ontology-free but requires training text, for which, they used text data from Mayo Clinic corpus of medical notes. 


\subsubsection{Nguyen and Al-Mubaid Measure}

Hisham Al-Mubaid \& Nguyen [14] [15] proposed measure takes the depth of their least common subsume (LCS) and the distance of the shortest path between them. The higher similarity arises when the two conceptsare in the lower level of the hierarchy. Their similarity measure is:

$$
\operatorname{Sim}(c 1, c 2)=\log \log 2([l(\mathrm{c} 1, \mathrm{c} 2)-1] \times[\operatorname{CSpec}(\mathrm{C} 1, \mathrm{C} 2)]+2)
$$

where:

$$
\mathrm{CSpec}(\mathrm{C} 1, \mathrm{C} 2)=\mathrm{D}-\operatorname{depth}(\mathrm{L}(\mathrm{c} 1, \mathrm{c} 2))
$$

Depth $\mathrm{L}(\mathrm{c} 1, \mathrm{c} 2)$ is depth of $\mathrm{L}(\mathrm{c} 1, \mathrm{c} 2)$ using node counting.

$\mathrm{L}(\mathrm{c} 1, \mathrm{c} 2)$ is the shortest distance between $\mathrm{c} 1$ and $\mathrm{c} 2$.

$\mathrm{D}$ is the maximum depth of the taxonomy.

The similarity equal 1, where two concept nodes are in the same cluster/ ontology. The maximum value of this measure occurs when one of the concepts is the left-most leaf node, and the other concept is a right leaf node in the tree.

Figure 2 shows the path length between "Cholera [A00]" and "Typhoid and paratyphoid fevers [A01]" is 3 using node counting. The path length between "Cholera due to Vibrio cholerae 01, biovarcholerae [A00.0]" and "Cholera due to Vibrio cholerae 01, biovareditor [A00.1]" is also 3. Thus, the similarity in these two cases is the same by Path length measure. However, the similarity between Cholera [A00]" and "Typhoid and paratyphoid fevers [A01]" is less than the similarity between "Cholera due to Vibrio cholerae 01, biovar cholerae [A00.0]" and "Cholera due to Vibrio cholerae 01, biovareltor [A00.1]" as the latter two concepts lie at a lower level in the hierarchy tree and share more information. However, Table 1 shows that Path length (P.L.), Wu \& Palmer, and Leacock \& Chodorow (L.C.) produce the same semantic similarity for the two pairs [(A00,

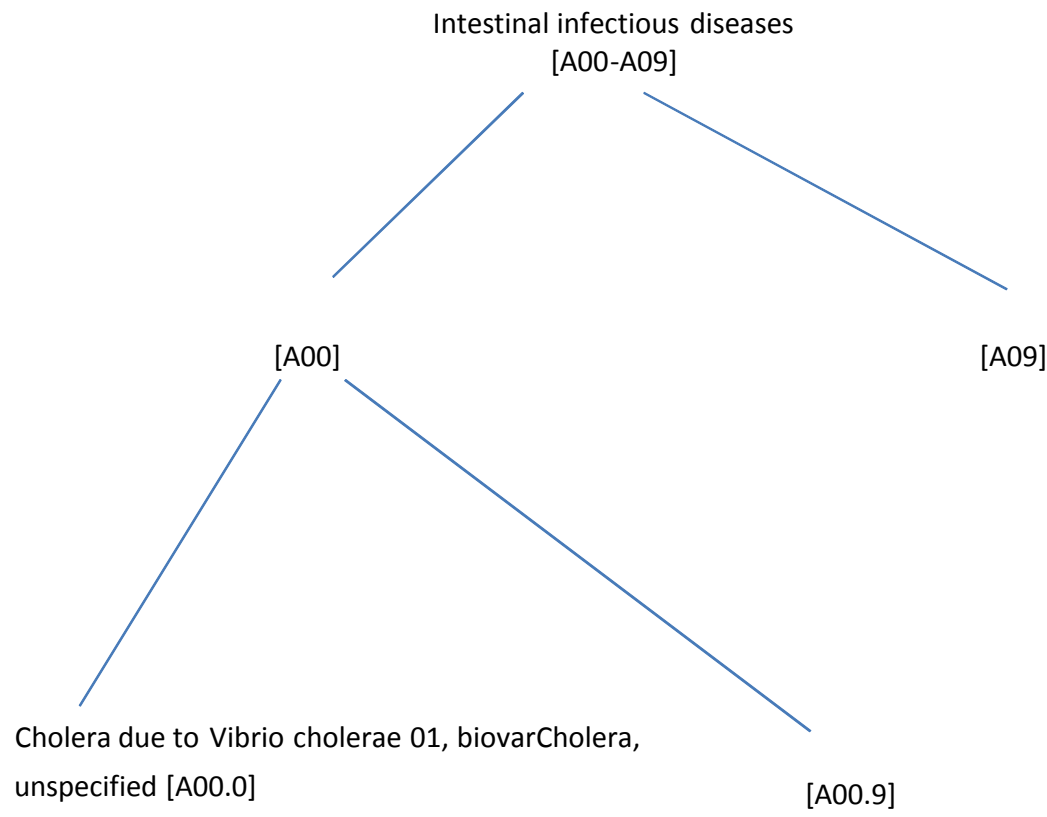

Figure 2. Fragment of ICD-10 ontology. 
Table 1. Measure comparison.

\begin{tabular}{ccccc}
\hline Pair of Concepts & P. $L$ & Wu \& Palmer & L. C & Al-Mubaid \& Nyguan Measure (Eq. (10)) \\
\hline A00 - A09 & 0.3 & 0.5 & 0.397 & 3.0 \\
A00.1 - A00.9 & 0.3 & 0.5 & 0.397 & 1.0 \\
\hline
\end{tabular}

A09) and (A00.1, A00.9)], whereas Al-Mubaid \& Nyguan measure gives a higher similarity (3.0) for the pair (A00.1, A00.9) as it occurs lower down in the ontology hierarchy than (A00.1, A00.9) which received the lower similarity (1.0). Recall that, in Al-Mubaid \& Nyguan Measure, Equation (10), the higher the numeric similarity result between $(\mathrm{c} 1, \mathrm{c} 2)$ the lower the semantic similarity between (c1, c2). In Wu \& Palmer measure, the path length between two concepts is not used, only depths of concepts are used, consequently, its performance is lower than Al-Mubaid \& Nyguan method [15].

$$
\begin{aligned}
& \operatorname{Sim}(\text { A00.0, A00.0 })=0 \text { then maximum similarity. } \\
& \operatorname{Sim}(\text { A00, A09 })=\log 2([3-1] \times[(5-2)])+2=3 . \\
& \operatorname{Sim}(\text { A00.1, A00.9) }=\log 2([4-1] \times(5-5))+2=1 .
\end{aligned}
$$

\section{Experiments and Results}

\subsection{Datasets}

In the biomedical domain, there are no standard human rating sets of terms/ concepts on semantic similarity and relatedness like the M \& C or R \& G sets for general English [16]. To comparemethods, we borrowed and used the set of 30 concept pairs from Pedersen, Pakhomov, \& Patwardhan (2005) [1], which was annotated by 3 physicians and 9 medical index experts. Each pair was annotated on a 4 point scale: "practically synonymous, related, marginally, and unrelated." The average correlation between physicians is 0.68 , and between experts is 0.78 .

In this paper, we examine only ontology-only techniques, and we use ICD10 the ontology instead of MeSH. We could find only 21 out of the 30 concept pairs in ICD10 using ICD10 browser ICD-10 Version: 2010

(http://apps.who.int/classifications/icd10/browse/2010/en) as some terms cannot be found, so we used 21 pairs in the experiments (Pedersen et al. [1] tested 29 out of the 30 concept pairs as one pair was not found in SNOMED-CT). The concept pairs in bold, in Table 2, are the ones that contain a term that was not found in ICD10 and we did not include in our experiments.

\subsection{Experiments and Results}

We implemented the Al-Mubaid \& Nyguan's similarity measure and conducted comparisons with four other ontology-based semantic similarity measures. All the measures use node counting for path length and for depth of concept nodes. For the pairs that have a term belongs to more than one category tree, we take into account only its position(s) in the same category with the other term. Table 3 shows for the five measures the results of correlation with human ratings of 
Table 2. The test set of 30 medical term pairs sorted in the order of the averaged physician's scores.

\begin{tabular}{|c|c|c|c|c|}
\hline Id & Concept 1 & Concept 2 & Phys & Expert \\
\hline 4 & Renal failure & Kidney failure & 4.0000 & 4.0000 \\
\hline 5 & Heart & Myocardium & 3.3333 & 3.0000 \\
\hline 1 & Stroke & Infarct & 3.0000 & 2.7778 \\
\hline 7 & Abortion & Miscarriage & 3.0000 & 3.3333 \\
\hline 9 & Delusion & Schizophrenia & 3.0000 & 2.2222 \\
\hline 11 & Congestive heart failure & Pulmonary edema & 3.0000 & 1.4444 \\
\hline 8 & Metastasis & Adenocarcinoma & 2.6667 & 1.7778 \\
\hline 17 & Calcification & Stenosis & 2.6667 & 2.0000 \\
\hline 10 & Diarrhea & Stomach cramps & 2.3333 & 1.3333 \\
\hline 19 & Mitral stenosis & Atrial fibrillation & 2.3333 & 1.3333 \\
\hline 20 & $\begin{array}{l}\text { Chronic obstructive } \\
\text { pulmonary disease }\end{array}$ & Lung infiltrates & 2.0000 & 1.8889 \\
\hline 2 & Rheumatoid arthritis & Lupus & 2.0000 & 1.1111 \\
\hline 3 & Brain tumor & Intracranial hemorrhage & 2.0000 & 1.3333 \\
\hline 15 & $\begin{array}{l}\text { Carpal tunnel } \\
\text { Syndrome }\end{array}$ & Osteoarthritis & 2.0000 & 1.1111 \\
\hline 18 & Diabetes mellitus & Hypertension & 2.0000 & 1.0000 \\
\hline 27 & Acne & Syringe & 2.0000 & 1.0000 \\
\hline 12 & Antibiotic & Allergy & 1.6667 & 1.2222 \\
\hline 13 & Cortisone & Total knee replacement & 1.6667 & 1.0000 \\
\hline 14 & Pulmonary embolus & Myocardial infarction & 1.6667 & 1.2222 \\
\hline 16 & Pulmonary Fibrosis & Lung Cancer & 1.6667 & 1.4444 \\
\hline 6 & Cholangiocarcinoma & Colonoscopy & 1.3333 & 1.0000 \\
\hline 29 & Lymphoid hyperplasia & Laryngeal Cancer & 1.3333 & 1.0000 \\
\hline 21 & Multiple Sclerosis & Psychosis & 1.0000 & 1.0000 \\
\hline 22 & Appendicitis & Osteoporosis & 1.0000 & 1.0000 \\
\hline 23 & Rectal polyp & Aorta & 1.0000 & 1.0000 \\
\hline 24 & Xerostomia & Alcoholic cirrhosis & 1.0000 & 1.0000 \\
\hline 25 & Peptic ulcer disease & Myopia & 1.0000 & 1.0000 \\
\hline 26 & Depression & Cellulitis & 1.0000 & 1.0000 \\
\hline 28 & Varicose vein & Entire knee meniscus & 1.0000 & 1.0000 \\
\hline 30 & Metastasis & Hyperlipidemia & 1.0000 & 1.0000 \\
\hline
\end{tabular}

Table 3. Absolute values of correlation of the five measures relative to human judgments.

\begin{tabular}{ccc}
\hline Measure & Physician (rank) & Expert (rank) \\
\hline Path length & $0.627(4)$ & $0.852(3)$ \\
Leacock \& Chodorow & $0.672(1)$ & $0.856(2)$ \\
Wu \& Palmer & $0.652(3)$ & $0.794(4)$ \\
Lin Measure & $0.560(5)$ & $0.724(5)$ \\
Al-Mubaid \& Nyguan's measure & $\mathbf{0 . 6 6 6 ( 2 )}$ & $\mathbf{0 . 8 6 2 ( 1 )}$
\end{tabular}


physicians and experts with the ranks between parentheses. These correlation values (in Table 3) show that Al-Mubaid \& Nyguan's method is ranked \#1 in correlation relative to experts' judgments. But relative to physician judgments, their method scored the second. Because the expert scores are more reliable as the correlation among the expert scores (0.78) is higher than that among the physicians (0.68), and there are more experts than physicians (3 physicians \& 9 experts).

\section{Conclusion and Future Works}

We have compared an ontology-based semantic similarity measure. The experiments presented in this paper have proven the superiority of the Al-Mubaid \& $N y g u a n ' s$ method relative to human judgments and compared with other ontology-based measures. In future work of this paper, we intend to explore experiment with applications of semantic relatedness measures to NLP tasks such as wordsense discrimination, information retrieval, and spelling correction, in the biomedical domain. We further use that set to compare taxonomies as well as calculate semantic similarity of two concepts within and across UMLS terminology sources. Finally, we plan to implement a web-based user interface for all these semantic similarity measures and to make it available freely to researchers over the Internet. That will be much helpful for interested researchers in the field of biomedical.

\section{References}

[1] Pedersen, T., et al. (2007) Measures of Semantic Similarity and Relatedness in the Biomedical Domain. Journal of Biomedical Informatics, 40, 288-299. https://doi.org/10.1016/j.jbi.2006.06.004

[2] Nguyen, H.A. (2006) New Semantic Similarity Techniques of Concepts Applied in the Biomedical Domain and WordNet. Master Thesis, The University of HoustonClear Lake.

[3] World Health Organization (2016) ICD-10, International Statistical Classification of Diseases and Related Health Problems. 5th Edition, Vol. 2.

[4] Dogaru, R., et al. (2015) Searching for Taxonomy-Based Similarity Measures for Medical Data. BCI, West University of Timisoara, 214.

[5] Rada, R., et al. (1989) Development and Application of a Metric on Semantic Nets. IEEE Transactions on Systems, Man, and Cybernetics, 17-30.

https://doi.org/10.1109/21.24528

[6] Meng, et al. (2013) A Review of Semantic Similarity Measures in WordNet. International Journal of Hybrid Information Technology, 1-12.

[7] Lin, D. (1993) Principle-Based Parsing without over Generation. Proceedings of the 31 st Annual Meeting of the Association for Computational Linguistics (ACL'93), Columbus, 112-120.

[8] Al-Mubaid, H. and Nguyen, H.A. (2006) A Cluster-Based Approach for Semantic Similarity in the Biomedical Domain. Proceedings of the 28th IEEE, EMBS Annual International Conference, New York, 30 Augugst-3 September 2006, 2713-2717.

[9] Batet, M., et al. (2011) An Ontology-Based Measure to Compute Semantic Similarity in Biomedicine. Journal of Biomedical Informatics, 44, 118-125. 
https://doi.org/10.1016/j.jbi.2010.09.002

[10] Anitha Elavarasi, S., et al. (2014) A Survey on Semantic Similarity Measure. International Journal of Research in Advent Technology, 2.

[11] Abdelrahman, A.M.B. and Kayed, A. (2015) A Survey on Semantic Similarity Measures between Concepts in Health Domain. American Journal of Computational Mathematics, 204.

[12] Thabet, T.S.S. (2013) Description and Evaluation of Semantic Similarity Measures Approaches. arXiv:1310.8059.

[13] Ensan, F. and Du, W.C. (2013) A Semantic Metrics Suite for Evaluating Modular Ontologies. Information Systems, 38, 745-770.

[14] Al-Mubaid, H. and Nguyen, H.A. (2009) Measuring Semantic Similarity between Biomedical Concepts within Multiple Ontologies. IEEE Transactions on Systems, Man, and Cybernetics-Part C: Applications and Reviews, 389-397. https://doi.org/10.1109/TSMCC.2009.2020689

[15] Nguyen, H.A. and Al-Mubaid, H. (2006) New Ontology-Based Semantic Similarity Measure for the Biomedical Domain. IEEE.

[16] Rubenstein, H. and Goodenough, J.B. (1965) Contextual Correlates of Synonymy. Communications of the ACM, 627-633.

Submit or recommend next manuscript to SCIRP and we will provide best service for you:

Accepting pre-submission inquiries through Email, Facebook, LinkedIn, Twitter, etc. A wide selection of journals (inclusive of 9 subjects, more than 200 journals) Providing 24-hour high-quality service User-friendly online submission system Fair and swift peer-review system Efficient typesetting and proofreading procedure Display of the result of downloads and visits, as well as the number of cited articles Maximum dissemination of your research work

Submit your manuscript at: http://papersubmission.scirp.org/

Or contact jcc@scirp.org 